\title{
Rocking-Beam Variable Coherence Electron Microscopy: An Alternative Approach to Fluctuation Electron Microscopy
}

\author{
J. Li* and I.M. Anderson**
}

* Metals and Ceramics Division, Oak Ridge National Laboratory, P.O. Box 2008, Oak Ridge, TN 37831-6116

**National Institute of Standards and Technology, 100 Bureau Dr., Gaithersburg, MD 20899-8371

Fluctuation electron microscopy (FEM) is a novel technique to characterize the "medium-range order" (MRO) in amorphous materials [1-2]. MRO is the order that extends beyond the second and third atomic shell $(\sim 1.5-2 \mathrm{~nm})$ but cannot be identified with high-resolution transmission electron microscopy (HRTEM). MRO is believed to affect diffusive, mechanical, optical and electronic properties of amorphous materials. FEM provides a measure of MRO through analysis of the normalized variance, $V(\boldsymbol{k}, Q)$, of the image intensity, $I(\boldsymbol{r})$, in a series of dark-field (DF) TEM images,

$$
V(k, Q)=\frac{\left\langle I^{2}(r, k, Q)\right\rangle}{\langle I(r, k, Q)\rangle^{2}}-1 \text {, }
$$

where the statistical average is over the real-space coordinate $\boldsymbol{r}$ in a given image, $\boldsymbol{k}$ is the scattering vector, and $Q$ is the diameter of the objective aperture at which the image was acquired [1-2]. Equivalently, the data may be acquired in scanning transmission electron microscopy (STEM) mode with a series of diffraction patterns, $I(\boldsymbol{k})$, with $Q$ representing the diameter of the probe-defining aperture [3]. In the typical case where the variance is measured as a function of $\boldsymbol{k}$ at constant $Q$, FEM is alternatively named variable coherence electron microscopy (VCEM). Traditionally, FEM is performed in TEM mode with annular hollow-cone dark field (HCDF) illumination to form an incoherent average image over all azimuths at a given $k=|\boldsymbol{k}|$ and a one-dimensional $V(k)$ profile.

We have been exploring an alternative data collection strategy for FEM, where a series of TEM DF images are acquired at distinct scattering vectors along a two-dimensional grid $\boldsymbol{k}=\left(k_{x}, k_{y}\right)$, as shown schematically in Fig. 1. This rocking beam (RB) VCEM can be usefully regarded as a fourdimensional extension of hyperspectral imaging, with two real-space and two reciprocal-space coordinates. We have found that the DF images formed in RB-VCEM mode have greater contrast than those acquired with HCDF illumination, which may help to explain the systematically lower values of $V(\boldsymbol{k}, Q)$ observed in TEM relative to those acquired in the equivalent STEM mode [3]. RBVCEM also provides advantages for study of MRO during the early stages of the amorphous-tocrystalline transition, since nanocrystalline precipitates can be identified by their characteristic spot diffraction patterns and removed prior to calculation of $V(\boldsymbol{k}, Q)$ for the adjacent amorphous matrix.

As a proof-of-principle, we have performed RB-VCEM on an amorphous tungsten (a-W) film sputtered onto a holey carbon grid. Series of DF images were acquired with a Philips CM200FEG $\mathrm{STEM} / \mathrm{TEM}$ operated at $200 \mathrm{kV}$ in "rocking beam" mode, $\sim 10 \mathrm{nA}$ beam current, "parallel" incident illumination, $135 \mathrm{kX}$ mag, and an objective aperture diameter $Q=\sim 2.4 \mathrm{~nm}^{-1}$. The beam orientation was varied manually on a 10x10 grid with a step size of $1 \mathrm{~nm}^{-\mathrm{T}}$ with an EMiSPEC Vision integrated acquisition system, in synchronicity with a Gatan slow-scan CCD camera, 2x binning, $10 \mathrm{~s}$ exposure operated in Acquire Series mode in DigitalMicrograph on a $15 \mathrm{~s}$ interval. The rocking-beam channeling pattern, formed with the STEM bright field detector centered on the optic axis, is shown in Fig. 1B. A false-color map of the corresponding $V\left(k_{x}, k_{y}\right)$ data is shown in Fig. 2. The peak in the $V(\boldsymbol{k})$ map at $k \sim 4.2 \mathrm{~nm}^{-1}$ and a value of $0.07-0.095$ coincides with the corresponding peak in the diffraction pattern. The influence of beam damage and energy filtering has also been studied [4]. 
[1] M.M.J. Treacy and J.M. Gibson, Acta Cryst. A 52 (1996) 212.

[2] J.M. Gibson et al., Ultramicrosc. 83 (2000) 169.

[3] P.M. Voyles and D.A. Muller, Ultramicrosc. 93 (2002) 147.

[4] Research at the SHaRE User Facility was sponsored by the Office of Basic Energy Sciences, U.S. Department of Energy, under contract DE-AC05-00OR22725 with UT-Battelle LLC.
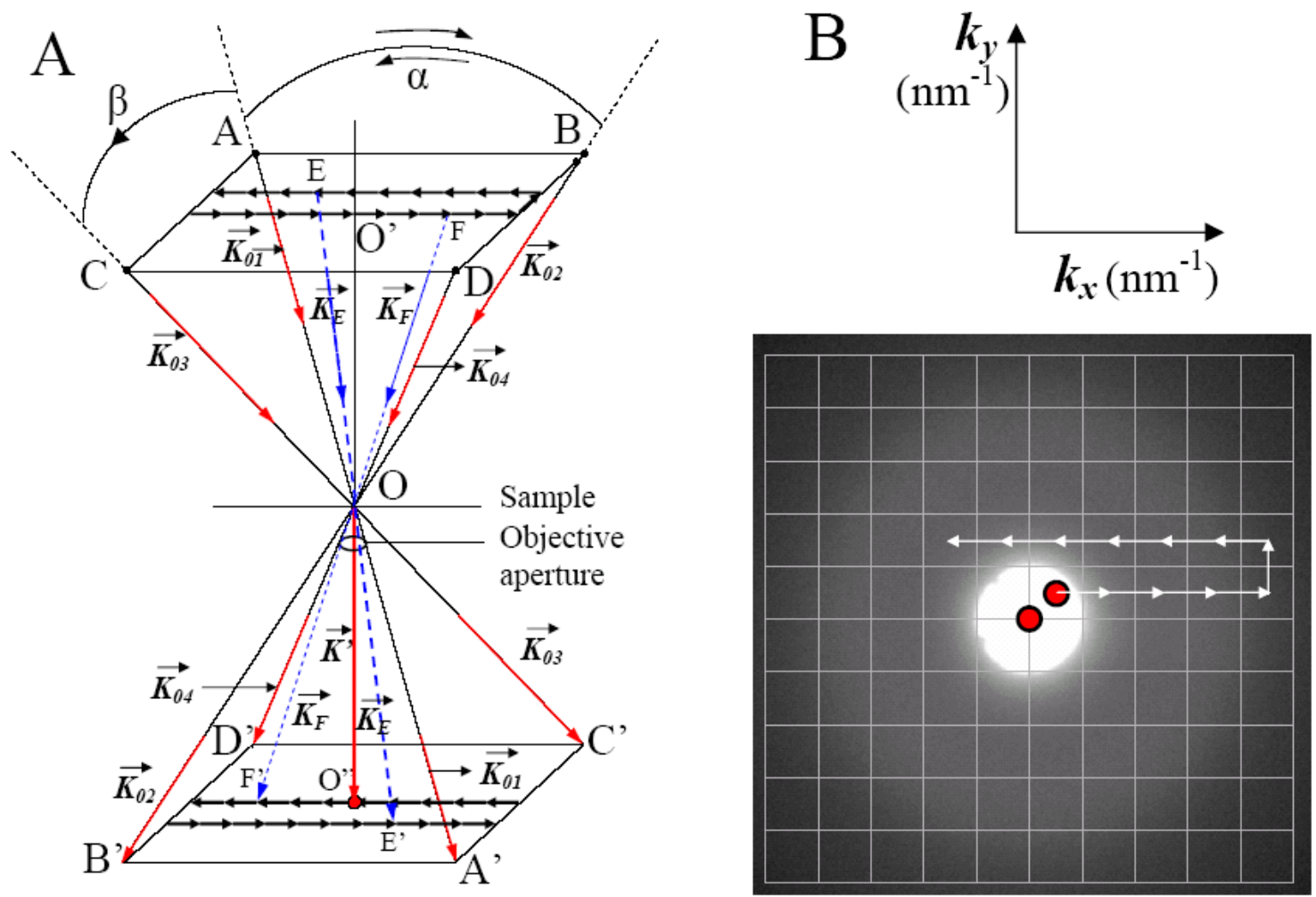

Fig. 1. A. Schematic representation of rocking-beam variable coherence electron microscopy (RBVCEM). Incident beam angles $\alpha$ and $\beta$ are varied in two dimensions to form a space defined by the "four-fold prism cone" ABDCO. $\boldsymbol{K}$ ' is the diffracted beam wave vector which is collected by a centered objective aperture to record TEM DF image. The blue arrows show two examples in TEM rocking-beam mode for the incident wave vectors $\boldsymbol{K}_{E}, \boldsymbol{K}_{F}$ along EOE', FOF' directions, respectively. B. Rocking-beam channeling pattern and grid over which RB-VCEM images were acquired.
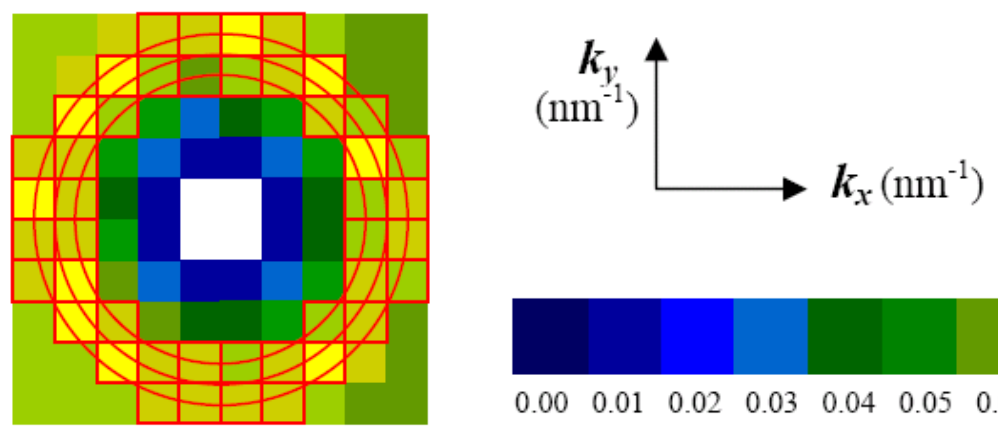

The variances have peak values of $0.07-0.095$ shown as a circular peak at the $(\boldsymbol{k} x, \boldsymbol{k} \boldsymbol{y})$ map with scattering vector $\boldsymbol{k} \sim 4.2 \mathrm{~nm}^{-1}$ which corresponds to the first halo in the amorphous W diffraction pattern.

Fig. 2. Variance map $V(\boldsymbol{k})$ of a-W as the function of two dimensional scattering vector $\boldsymbol{k}=\left(k_{x}, k_{y}\right)$. 